\title{
Patient-reported outcomes after surgery or radiotherapy for localised prostate cancer: a retrospective study
}

\author{
CF Ng *, KY Kong, CY Li, Jennifer KT Li, NY Li, Brian PK Ng, Steven CH Leung, Cindy YL Hong, \\ $\mathrm{CH}$ Yee, Jeremy YC Teoh
}

\section{A B S T R A C T}

Introduction: To compare the intermediate-term outcomes and patient-reported outcomes of robotassisted laparoscopic prostatectomy (RALP) and radical external beam radiotherapy (RT) in Chinese patients with localised prostate cancer.

Methods: This was a retrospective study of patients with localised prostate cancer diagnosed between 2010 and 2011 and treated with either RALP or RT. Baseline patient and disease characteristics, posttreatment complications, and latest disease status were retrospectively collected from hospital records. For assessment of patient-reported outcomes, the Chinese version of the Expanded Prostate Cancer Index Composite (EPIC) questionnaire was completed by the patients.

Results: Ninety three patients aged 58 to 84 years were recruited. Thirty patients were treated by RALP (32.3\%), whereas 63 received RT (67.7\%). The RALP group had significantly lower baseline prostatespecific antigen levels than the RT group $(\mathrm{P}<0.001)$. More patients who underwent RALP reported urinary incontinence $(70.0 \%$ vs $3.2 \%, \quad \mathrm{P}<0.001)$, whereas more patients who underwent RT reported other voiding symptoms $(87.3 \%$ vs $50.0 \%, \mathrm{P}<0.001)$ and perirectal bleeding $(36.5 \%$ vs $0 \%, \mathrm{P}<0.001)$ during follow-up. Of the 85 patients who were still alive at the time of the study, $52(61.2 \%)$ returned completed questionnaires. Patients who underwent
EPIC urinary summary scores than patients who underwent RT [81.5 (18.3) vs 88.9 (17.9), $\mathrm{P}=0.016$ ]. Urinary function [75.9 (20.4) vs 93.6 (16.2), $\mathrm{P}<0.001]$ and incontinence [60.5 (31.8) vs 91.8 (14.5), $\mathrm{P}<0.001]$ were also significantly worse in the RALP group. The bowel and sexual domain scores were similar between the two groups.

Conclusions: We found that RALP and RT were associated with different patterns of complications and patient-reported outcomes. Urinary incontinence was much more prevalent in the patients treated surgically. This may significantly affect patients' quality of life.

\section{Hong Kong Med J 2020;26:95-101 \\ https://doi.org/10.12809/hkmj198239}

CF Ng *, MB, ChB, FHKAM (Surgery)

KY Kong, MB, ChB

CY Li, MB, ChB

JKT Li, MB, ChB

NY Li, MB, ChB

BPK Ng, MB, ChB

SCH Leung, MSC

CYL Hong, MSc

CH Yee, MB, ChB, FHKAM (Surgery)

JYC Teoh, MB, ChB, FHKAM (Surgery)

SH Ho Urology Centre, Division of Urology, Department of Surgery, The Chinese University of Hong Kong, Shatin, Hong Kong

RALP had poorer median (interquartile range) * Corresponding author: ngcf@surgery.cuhk.edu.hk

New knowledge added by this study

- Patient-reported outcomes of treatment for localised prostate cancer in a Chinese cohort

- Intermediate-term outcomes and complications experienced by patients, from physician assessments

- Relationship between unplanned hospitalisation and treatment

Implications for clinical practice or policy

- Potential combined use of patient-reported outcomes of treatment and physician consultations

- Consideration of treatment based on complications

\section{Introduction}

In 2016, prostate cancer was the third most common type of cancer in men in Hong Kong, with 1912 new cases diagnosed. ${ }^{1}$ Increased disease awareness and the use of prostate-specific antigen (PSA) screening have increased the frequency of diagnosis of earlystage prostate cancers. ${ }^{2}$
While active surveillance has become increasingly common for low-risk localised prostate cancer, robot-assisted laparoscopic prostatectomy (RALP) and external beam radiotherapy (RT) remain the mainstay treatments for localised prostate cancer. Despite the effectiveness of both treatments, treatment-related adverse effects are not uncommon. 


\section{局部前列腺癌手術或放療後的患者報告結果： 回顧研究 \\ 吳志輝、江嘉愉、李靜欣、李幗婷、李雅欣、吳寶鈞、 梁志豪、匡若琳、余知行、張源津}

引言：比較機械臂輔助腹腔鏡前列腺切除術 (RALP) 和體外放療 （RT）對華籍局部前列腺癌患者的中期結果和患者報告結果。

方法：這項回顧研究包括 2010 年至 2011 年間經診斷後接受RALP或RT 的局部前列腺癌患者。從醫院記錄收集患者的基線和疾病特徵、治療 後併發症以及最新疾病狀態的信息。患者須填寫擴展前列腺癌綜合指 數問卷中文版（EPIC）以評估其患者報告結果。

結果：研究納入93名58至84歲患者。30例（32.3\%）接受RALP， 63 例 $(67.7 \%$ ) 接受RT。RALP組的基線前列腺特異性抗原水平明顯 低於RT組 $(\mathrm{P}<0.001)$ RALP組報告尿失禁的比例較高（ $70.0 \%$ 比 $3.2 \% ， \mathrm{P}<0.001 ）$ ，而較高比例的RT患者報告其他排尿症狀（ $87.3 \%$ 比 $50.0 \%, \mathrm{P}<0.001)$ 和直腸圍出血（36.5\%比 $0 \%, \mathrm{P}<0.001 ） 。$ 進 行研究時還存活的 85 名患者中, 52 名 $(61.2 \%)$ 交回完整問卷。與 RT組相比, RALP組的EPIC尿液綜合評分較差（中位數81.5〔四分 位距18.3〕比88.9〔17.9〕， $\mathrm{P}=0.016) 。 \mathrm{RALP}$ 組的尿功能（75.9 〔20.4〕比93.6〔 16.2〕， $\mathrm{P}<0.001)$ 和尿失禁（60.5〔31.8〕比91.8 〔 14.5〕， P < 0.001) 也明顯較差。兩組的腸和性結構得分相若。

結論：研究顯示RALP和RT與各種併發症和患者報告結果相關。尿失 禁在外科治療的患者中更為普遍, 或會對患者的生活質量造成嚴重影 響。
Cluster Clinical Research Ethics Committee, Ref CREC 2016.373) and conducted according to the Declaration of Helsinki.

Patients' demographic characteristics, pretreatment disease characteristics, treatment outcomes, and complications were retrieved from hospital records. The patients' quality of life was assessed using a validated PRO measure, the Expanded Prostate Cancer Index Composite (EPIC) questionnaire. ${ }^{6}$ The EPIC questionnaire has been validated as a measure of disease-specific function. The full version of the EPIC questionnaire consists of 50 questions divided into four treatment complication-related domains: urinary symptoms, bowel symptoms, sexual symptoms, and hormonal treatment-related symptoms. Each domain is further subdivided into different functional components that evaluate the severity of adverse effects and level of bother (ie, distress) related to the symptoms and adverse effects. Most of the questions are scored on a Likert scale, with the majority using a 5-point scale. The calculation of final quality of life was based on the EPIC scoring guideline; the final score ranged from 0 to 100, with higher scores representing better quality of life. A validated Chinese version of this questionnaire was used. ${ }^{7}$ Copies of the questionnaire were mailed to the patients' home addresses in early December 2016, and the patients were given 2 weeks to complete the questionnaire. Patients who participated in the PRO segment consented to their participation in the study.

All of the statistical analyses were performed using IBM SPSS Statistics (Windows version 25.0; IBM Corp., Armonk [NY], United States). Personnel involved in the collection and input of EPIC data were blinded to the patients' clinical information. The results were deemed statistically significant at a $\mathrm{P}$ value of $<0.05$. The results for the RALP and RT groups were compared using the Chi squared test, Fisher's exact test, or Student's $t$ test. The EPIC scores for the two treatment arms were compared using the Mann-Whitney $U$ test. The details of individual symptoms and their impact on quality of life were also assessed. Missing values were excluded from analysis.

\section{Results}

\section{Patients' baseline characteristics}

Ninety three men aged 58 to 84 years were recruited to take part in the study. Eighty five of these patients were still alive during the study period, but eight died due to non-prostate cancer-related causes (RALP $n=1$, RT $n=7)$. Thirty (32.3\%) patients had been treated by RALP, whereas $63(67.7 \%)$ had received RT. Fifty two patients returned the questionnaires, giving a response rate of $61.2 \%$ (Table 1 ).

The mean ages of the patients who underwent RALP and RT were $65.8 \pm 6.07$ and $69.81 \pm 5.48$ years, 
respectively, and the RALP patients were significantly younger than the RT patients $(\mathrm{P}=0.002)$. More than half of the patients in each group were defined as Eastern Cooperative Oncology Group category 1.

The pretreatment serum PSA levels of the patients who underwent RALP $(9.6 \pm 4.18 \mathrm{ng} / \mathrm{mL})$ were also significantly lower than those of the patients who underwent RT $(29.44 \pm 32.03 \mathrm{ng} / \mathrm{mL}$, $\mathrm{P}<0.001)$. One third of the patients in the RT $(33.3 \%)$ and half of the patients in the RALP (50.0\%) group were diagnosed as T1c. Sixty percent of the patients who underwent RALP and $38.1 \%$ of the patients who underwent RT had Gleason scores <7; only $23.3 \%$ of the RALP patients and $36.5 \%$ of the RT patients had scores $>7$. The Gleason scores for the patients who underwent RALP were lower than those for the patients who underwent $\mathrm{RT}$, although the difference was not statistically significant $(\mathrm{P}=0.185)$. There was a trend of more patients with D'Amico high-risk cancer in the RT group than the RALP group (Table 2).

\section{Treatment outcomes}

All patients who underwent RALP achieved undetectable serum PSA levels after surgery, whereas $46.0 \%$ of the patients who underwent RT had undetectable PSA levels. During the follow-up period, 12 (19.0\%) patients from the RT group and two $(6.7 \%)$ patients from the RALP group developed biochemical recurrence, but this difference was statistically insignificant $(P=0.213)$. The majority of the patients who underwent RT and had biochemical recurrence $(75 \%)$ opted for androgen deprivation therapy for further treatment, whereas all RALP patients with biochemical recurrence (100\%) chose salvage RT as an additional therapy. About $5 \%$ of the patients in the RT group developed metastatic disease during the follow-up period, while none in the RALP group did so (Table 3).

\section{Complications}

The clinical information indicates that the majority of the patients in both groups suffered urinary symptoms to some degree during the first 3 months after treatment. However, short-term bowel dysfunction was more prevalent in the patients who underwent RT (46\%) than in the patients who underwent RALP $(6.7 \% ; \mathrm{P}<0.001)$.

Regarding intermediate-term complications reported during physician consultation, $70 \%$ of the RALP group patients reported urinary incontinence, compared with only $3.2 \%$ of the RT group patients $(\mathrm{P}<0.001)$. In contrast, more RT group patients (87.3\%) than RALP group patients (50\%) experienced lower urinary tract symptoms other than urinary incontinence $(\mathrm{P}<0.001)$. Furthermore, $36.5 \%$ of the RT group patients complained of perirectal bleeding, which was not reported by the RALP group patients
TABLE I. Demographics at diagnosis for 93 Chinese men with localised prostate cancer treated by radiotherapy or robot-assisted laparoscopic prostatectomy*

\begin{tabular}{lccc}
\hline & $\begin{array}{c}\text { Radiotherapy } \\
(\mathbf{n = 6 3 )}\end{array}$ & $\begin{array}{c}\text { Prostatectomy } \\
(\mathbf{n = 3 0 )}\end{array}$ & P value \\
\hline Age (years) & $69.81 \pm 5.48$ & $65.8 \pm 6.07$ & 0.002 \\
Follow-up time (months) & $69.75 \pm 11.53$ & $73.47 \pm 8.25$ & 0.117 \\
ECOG performance status & & & $0.005 \dagger$ \\
1 & $32(50.8 \%)$ & $25(83.3 \%)$ & \\
2 & $29(46.0 \%)$ & $5(16.7 \%)$ & \\
3 & $2(3.2 \%)$ & 0 & \\
\hline
\end{tabular}

Abbreviation: ECOG = Eastern Cooperative Oncology Group

* Data are shown as No. (\%) or mean \pm standard deviation, unless otherwise specified † Fisher's exact test

TABLE 2. Tumour characteristics and treatment outcomes of 93 Chinese men with localised prostate cancer treated by radiotherapy or robot-assisted laparoscopic prostatectomy*

\begin{tabular}{|c|c|c|c|}
\hline & $\begin{array}{l}\text { Radiotherapy } \\
(n=63)\end{array}$ & $\begin{array}{l}\text { Prostatectomy } \\
(n=30)\end{array}$ & $P$ value \\
\hline Pretreatment PSA (ng/mL) & $29.44 \pm 32.03$ & $9.6 \pm 4.18$ & $<0.001$ \\
\hline \multicolumn{4}{|l|}{ PSA grouping } \\
\hline $4-10 \mathrm{ng} / \mathrm{mL}$ & $15(23.8 \%)$ & 15 (50.0\%) & \\
\hline $10-20 \mathrm{ng} / \mathrm{mL}$ & $23(36.5 \%)$ & $14(46.7 \%)$ & \\
\hline$>20 \mathrm{ng} / \mathrm{mL}$ & $25(39.7 \%)$ & $1(3.3 \%)$ & \\
\hline Clinical stage & & & $0.077 \dagger$ \\
\hline $\mathrm{T} 1$ & $21(33.3 \%)$ & $15(50.0 \%)$ & \\
\hline $\mathrm{T} 2$ & $21(33.3 \%)$ & $12(40.0 \%)$ & \\
\hline T3 & $20(31.7 \%)$ & $3(10.0 \%)$ & \\
\hline $\mathrm{T} 4$ & $1(1.6 \%)$ & 0 & \\
\hline Gleason score & & & $0.185 \dagger$ \\
\hline$<7$ & $24(38.1 \%)$ & $18(60.0 \%)$ & \\
\hline $7(3+4)$ & $12(19.0 \%)$ & $5(16.7 \%)$ & \\
\hline $7(4+3)$ & $4(6.3 \%)$ & 0 & \\
\hline$>7$ & $23(36.5 \%)$ & $7(23.3 \%)$ & \\
\hline \multicolumn{4}{|l|}{ D'Amico classification } \\
\hline Low risk & $8(12.7 \%)$ & $8(26.7 \%)$ & $0.082 \ddagger$ \\
\hline Intermediate risk & $15(23.8 \%)$ & $10(33.3 \%)$ & \\
\hline High risk & $40(63.5 \%)$ & $12(40.0 \%)$ & \\
\hline
\end{tabular}

Abbreviation: PSA = prostate-specific antigen

* Data are shown as No. (\%) or mean \pm standard deviation, unless otherwise specified + Fisher's exact test

‡ Chi squared test

$(\mathrm{P}<0.001)$. Erectile dysfunction was more prevalent in the RALP group (85.2\%) than in the RT group (23.2\%), with $60.9 \%$ of the RALP group patients requesting treatment for this condition $(\mathrm{P}<0.001)$.

Six $(9.5 \%)$ of the patients who underwent RT had unplanned hospitalisations related to their 
TABLE 3. Treatment outcomes and complications of 93 Chinese men with localised prostate cancer treated by radiotherapy or robot-assisted laparoscopic prostatectomy*

\begin{tabular}{|c|c|c|c|}
\hline & $\begin{array}{l}\text { Radiotherapy } \\
\qquad(\mathrm{n}=63)\end{array}$ & $\begin{array}{l}\text { Prostatectomy } \\
\qquad(n=30)\end{array}$ & P value \\
\hline Use of ADT during primary therapy & $43(68.3 \%)$ & 0 & $<0.001$ \\
\hline Post-treatment PSA undetectable & $29(46.0 \%)$ & $30(100.0 \%)$ & $<0.001$ \\
\hline Biochemical recurrence & $12(19.0 \%)$ & $2(6.7 \%)$ & 0.213 \\
\hline Use of ADT during follow-up & $9(14.3 \%)$ & 0 & 0.054 \\
\hline Metastasis & $3(4.8 \%)$ & 0 & 0.548 \\
\hline Death & $7(11.1 \%)$ & $1(3.3 \%)$ & 0.430 \\
\hline \multicolumn{4}{|l|}{$\begin{array}{l}\text { Short-term complications } \\
\text { ( } \leq 3 \text { months after primary treatment) }\end{array}$} \\
\hline Urinary symptoms & $58(92.1 \%)$ & $28(93.3 \%)$ & 1.00 \\
\hline Bowel symptoms & $29(46.0 \%)$ & $2(6.7 \%)$ & $<0.001$ \\
\hline Skin rash & $3(4.8 \%)$ & $1(3.3 \%)$ & 1.00 \\
\hline \multicolumn{4}{|l|}{$\begin{array}{l}\text { Longer-term complications } \\
\text { (>3 months after primary treatment) }\end{array}$} \\
\hline Erectile dysfunction $\ddagger$ & $13 / 56(23.2 \%)$ & $23 / 27(85.2 \%)$ & $<0.001$ \\
\hline $\begin{array}{l}\text { Requested treatment for } \\
\text { erectile dysfunction }\end{array}$ & $2(15.4 \%)$ & $14(60.9 \%)$ & $<0.001$ \\
\hline Incontinence & $2(3.2 \%)$ & $21(70.0 \%)$ & $<0.001$ \\
\hline LUTS other than incontinence & $55(87.3 \%)$ & $15(50.0 \%)$ & $<0.001$ \\
\hline Haematuria & $4(6.3 \%)$ & $2(6.7 \%)$ & 1.00 \\
\hline Perirectal bleeding & $23(36.5 \%)$ & 0 & $<0.001$ \\
\hline Diarrhoea & $5(7.9 \%)$ & 0 & 0.171 \\
\hline Unplanned hospitalisation & $6(9.5 \%)$ & 0 & 0.172 \\
\hline
\end{tabular}

Abbreviations: ADT = androgen deprivation therapy; LUTS = lower urinary tract symptoms; PSA = prostate-specific antigen

* Data are shown as No (\%), unless otherwise specified

$\dagger$ Fisher's exact test

‡ Missing data and patients with erectile dysfunction before therapy were excluded prostate cancer or its treatment during the follow-up period. Four were due to haematuria, one was due to rectal bleeding, and one was due to acute urine retention. No unplanned hospital admissions were observed in the RALP group (Table 3 ).

\section{Results of Expanded Prostate Index Composite questionnaire}

The EPIC questionnaire was used to assess the patients' reported outcomes with regard to their choice of treatment and their urinary, bowel, and sexual function over the 4 weeks before the survey (Table 4 ). The overall response rate was $61.2 \%$ (52 out of 85 patients), and the response rates for the RT group (33 out of $56,58.9 \%$ ) and the RALP group (19 out of $29,65.5 \%)$ were similar.

The RALP group patients had poorer median (interquartile range) EPIC urinary summary scores (81.5 [18.3]) than the RT group patients had (88.9 [17.9]; $\quad \mathrm{P}=0.016)$. Significantly poorer urinary function and incontinence results were observed in the RALP group (75.9 [20.4] and 60.5 [31.8], respectively] than in the RT group (93.6 [16.2] and 91.8 [14.5], respectively) [both $\mathrm{P}<0.001]$. However, the two groups reported similar EPIC scores for urinary bother and urinary irritation/obstruction (Table 4 and Fig 1).

The patients who underwent both RT and RALP reported good bowel function, with median EPIC bowel summary scores of 92.9 (15.6) and 92.0 (19.6), respectively. There was no statistically significant difference between the two groups in terms of either bowel function or bowel bother (Fig 2).

TABLE 4. EPIC scores of 93 Chinese men with localised prostate cancer treated by radiotherapy or robot-assisted laparoscopic prostatectomy

\begin{tabular}{|c|c|c|c|c|c|}
\hline & \multicolumn{2}{|c|}{ Radiotherapy } & \multicolumn{2}{|c|}{ Prostatectomy } & \multirow[t]{2}{*}{$P$ value } \\
\hline & No. of patients & Median (IQR) & No. of patients & Median (IQR) & \\
\hline \multicolumn{6}{|l|}{ Urinary } \\
\hline Summary & 27 & $88.9(77.3-95.2)$ & 18 & $81.5(68.6-86.8)$ & 0.016 \\
\hline Function & 32 & $93.6(83.8-100.0)$ & 18 & $75.9(65.5-85.9)$ & $<0.001$ \\
\hline Bother & 28 & $82.1(71.4-92.9)$ & 19 & $75(60.7-92.9)$ & 0.366 \\
\hline Incontinence & 27 & $91.8(85.5-100.0)$ & 18 & $60.5(49.1-80.8)$ & $<0.001$ \\
\hline Irritative/obstructive symptoms & 27 & 85.7 (71.4-92.9) & 18 & $87.5(80.4-96.4)$ & 0.230 \\
\hline \multicolumn{6}{|l|}{ Bowel } \\
\hline Summary & 26 & $92.9(83-98.7)$ & 16 & $92.0(79.9-99.6)$ & 0.774 \\
\hline Function & 33 & $95.8(80.4-100.0)$ & 18 & $89.3(76.8-97.3)$ & 0.389 \\
\hline Bother & 26 & $96.4(78.6-100.0)$ & 17 & $92.9(82.1-100)$ & 0.918 \\
\hline \multicolumn{6}{|l|}{ Sexual } \\
\hline Summary & 30 & $22.4(7.7-30.8)$ & 16 & $31.7(9.8-42)$ & 0.092 \\
\hline Function & 29 & $2.8(0.0-13.4)$ & 16 & $10.6(0.7-32.8)$ & 0.097 \\
\hline Bother & 28 & $43.8(20.3-93.8)$ & 17 & $50.0(25.0-100.0)$ & 0.794 \\
\hline
\end{tabular}

Abbreviations: EPIC = Expanded Prostate Cancer Index Composite; IQR = interquartile range 


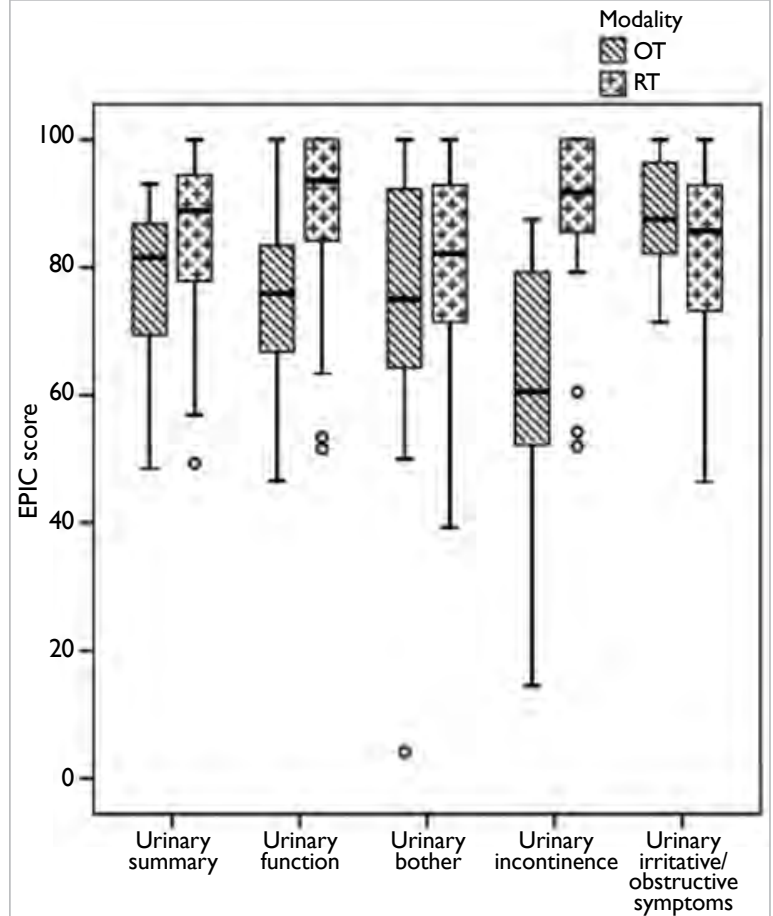

FIG I. EPIC scores, urinary domain, of 93 Chinese men with localised prostate cancer treated by radiotherapy or robotassisted laparoscopic prostatectomy

Abbreviations: EPIC = Expanded Prostate Cancer Index

Composite; OT = operative therapy; RT = radiotherapy

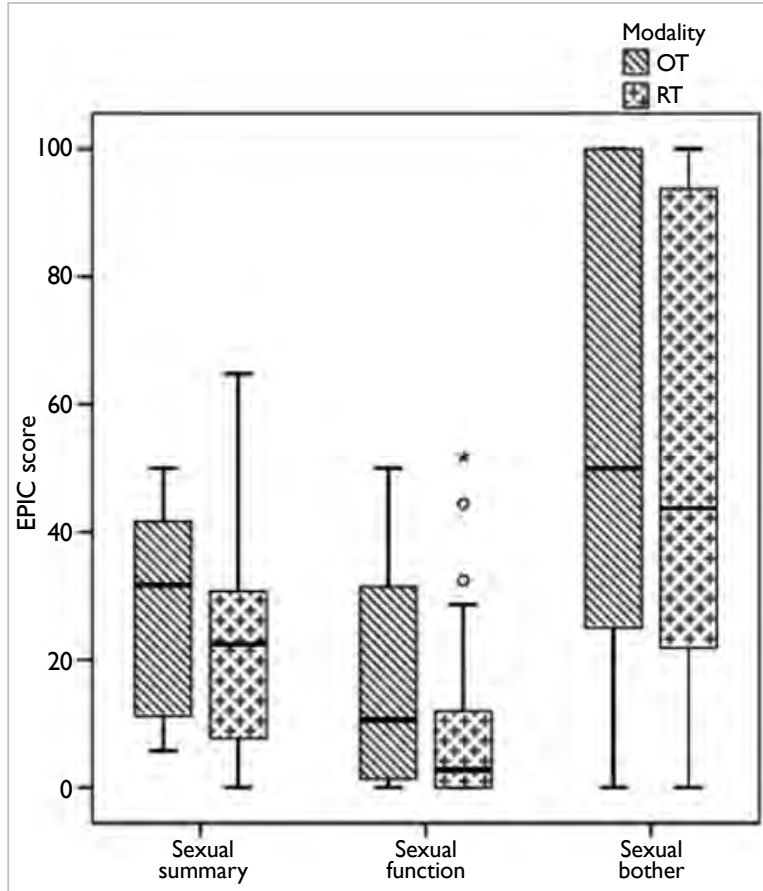

FIG 3. EPIC scores, sexual domain, of 93 Chinese men with localised prostate cancer treated by radiotherapy or robotassisted laparoscopic prostatectomy

Abbreviations: EPIC = Expanded Prostate Cancer Index Composite; OT = operative therapy; RT = radiotherapy

Poor median EPIC sexual summary and function scores were reported by both the RT (22.4 [23.1] and 2.8 [13.4], respectively) and the RALP (31.7 [32.2] and 10.6 [32.1], respectively) groups. Although low scores on the sexual bother subscale were also reported, they were less poor than those for the function subscale. However, no significant differences were observed between the RT and RALP groups on any of the sexual function scales (Fig 3).

\section{Discussion}

This study was the first to investigate the PROs of treatment for localised prostate cancer in a Chinese cohort and the intermediate-term complications experienced by patients. As expected, the complication profiles and PROs differed between patients who underwent radical prostatectomy and those who received external beam RT as treatment for localised prostate cancer. Compared with the RT group, the RALP group had less bowel disturbance immediately after treatment and less rectal bleeding during follow-up. The RALP group reported more urinary incontinence during follow-up, whereas the RT group experienced a greater frequency of other urinary symptoms. In addition, more unplanned admissions (mainly due to haematuria and rectal bleeding) were observed among the RT patients. 
However, the PRO results showed that the two groups achieved similar scores in other domains, except that the patients who underwent RALP had significantly lower incontinence and function scores.

Prostate cancer is the second most commonly diagnosed cancer in men in the world and the fifth leading cause of death from cancer in men. ${ }^{8}$ Although prostate cancer has a lower overall incidence in Asia than in Western countries, its incidence in Asia is rising. ${ }^{9}$ The rapid increase in incidence has been partly related to the increased usage of serum PSA for early cancer detection, which has resulted in increased detection of earlier-stage cancers. As a result, more patients can receive treatment with curative intent. This may be one cause of the decreasing prostate cancer mortality rate in many countries. $^{8,9}$

Currently, most Asian patients with localised prostate cancer choose active intervention as treatment; active surveillance is not commonly practised in the region. ${ }^{10}$ The two most commonly performed treatments for prostate cancer in Asia are RT and RALP. Therefore, our study focused on assessing the outcomes of these two treatment modalities. As these two treatments have similar oncological outcomes for localised cancer, ${ }^{11}$ the choice of treatment depends on other factors, such as the risk to patients associated with surgery and possible post-treatment complications. We also noticed that patients' age and performance status may affect the choice of treatment, as in our cohort, older patients and those with more co-morbidities were more likely to choose RT than surgery. Potential treatment-related complications were also a major consideration during the decision making process.

Some cross-sectional ${ }^{12}$ and prospective ${ }^{3,13}$ studies have investigated the effects of different types of treatment on the PROs of patients with localised prostate cancer. In general, these studies have found that different treatments are associated with different patterns of complications. Compared with RT, RALP has been shown to have greater negative effects on sexual function and urinary incontinence, whereas RT is associated with more bowel symptoms, especially bloody stools. Interestingly, sexual function has been found to gradually decline even under active surveillance, which is related to the natural ageing process. ${ }^{3}$ Our results indicated similar patterns of complications, with more RALP group patients reporting urinary incontinence and more RT group patients reporting bloody stools during follow-up. Similar to the results of the ProtecT study, ${ }^{4}$ we found that by around 6 years $(72$ months) after treatment, the two treatment groups achieved similar scores for voiding symptoms, bowel bother, and bowel function. In terms of sexual function, although both treatment groups had low sexual summary and function scores, the RT group generally had lower scores than the RALP group, although the difference was statistically insignificant. This finding was inconsistent with the results of previous studies. ${ }^{4}$ However, the higher mean age and greater reported use of hormonal therapy of our RT cohort compared with our RALP cohort may have resulted in poorer sexual function. Nevertheless, the two groups' sexual bother scores were similar, which suggests that the poorer sexual function reported by the RT group compared with the RALP group did not cause more bother to the patients.

There is little information available about the differences in PROs yielded by different modes of treatment for localised prostate cancer in Asian populations. Only one early report has been conducted with a Japanese population, in which urinary domain scores initially worsened after prostatectomy and brachytherapy, gradually improving later. ${ }^{14}$ Compared with other treatment modalities, external beam RT resulted in significantly lower bowel summary scores. Therefore, our study provides important information about the outcomes of treatment of localised prostate cancer, particularly the use of a relatively new treatment modality, RALP. More prospective multicentre studies would be helpful to provide more information to support patient counselling.

We not only investigated PROs, but also recorded symptoms/complications after treatment (ie, physician-reported outcomes) and unplanned admissions due to disease- or treatment-related complications. Although some studies have suggested that PROs are more accurate than physician-reported outcomes, ${ }^{15}$ we believed that combining the two would provide a more comprehensive picture of patients' post-treatment course. Our findings revealed not only differences in the patterns of urinary and bowel symptoms experienced by the two groups of patients, but also a trend of more unplanned hospital admissions in the RT group than the RALP group. Most of these admissions were related to the adverse effects of RT, such as haematuria and perirectal bleeding. Along with PROs, this information is important for patient counselling.

The study had several limitations. The sample was relatively small and involved only local Chinese participants. Currently, data on PROs for the local population are lacking. We hope our data can help to provide some information for patients and physicians during their decision making process. Our results were quite similar to the reported PROs for localised prostate cancer in the literature. Further, Tyson et $\mathrm{al}^{16}$ suggested there is not much racial difference in PROs amongst patients with prostate cancer. Second, as data on treatment-related complications were collected retrospectively, the information about the incidence of complications may have been 
biased, particularly for erectile dysfunction. Further prospective studies should provide more information on this topic. Furthermore, the number of patients was unbalanced between the two groups, as more RT-treated patients were recruited. This may have reflected the clinicians' and patients' preference. In addition, baseline disease characteristics differed between the two groups, with more high-risk patients in the RT group than in the RALP group. Therefore, oncological outcomes were not analysed further in this study. Finally, as active surveillance has only become common clinical practice in recent years, the number of patients receiving this treatment a few years ago was small. Therefore, we did not include this treatment modality in our initial planning, and no information about this treatment was available for comparison with findings for the other treatments.

\section{Conclusion}

Robot-assisted laparoscopic prostatectomy and external beam RT are associated with different patterns of complications and PROs with respect to urinary, bowel, and sexual function. Our results provide valuable information for counselling patients regarding treatment choices and outcome expectations.

\section{Author contributions}

Concept or design: CF Ng.

Acquisition of data: KY Kong, CY Li, JKT Li, NY Li, BPK Ng. Analysis or interpretation of data: KY Kong, CY Li, JKT Li, NY Li, BPK Ng, SCH Leung.

Drafting of the manuscript: CF Ng, SCH Leung.

Critical revision of the manuscript for important intellectual content: All authors.

All authors had full access to the data, contributed to the study, approved the final version for publication, and take responsibility for its accuracy and integrity.

\section{Conflicts of interest}

As editors of the journal, CF Ng and JYC Teoh were not involved in the peer review process. Other authors have disclosed no conflicts of interest.

\section{Funding/support}

This research received no specific grant from any funding agency in the public, commercial, or not-for-profit sectors.

\section{Ethics approval}

The study was conducted in accordance with good clinical practice guidelines and the Declaration of Helsinki. Ethics approval was granted by the Joint Chinese University of Hong Kong-New Territories East Cluster Clinical Research Ethics Committee (Ref CREC 2016.373). Informed consent was obtained from the patients when they completed the questionnaires.

\section{References}

1. Hong Kong Cancer Registry, Hospital Authority, Hong Kong SAR Government. Available from: http://www3. ha.org.hk/cancereg/pdf/factsheet/2016/prostate_2016. pdf. Accessed 6 Sep 2019.

2. Wong HF, Yee $\mathrm{CH}$, Teoh JY, et al. Time trend and characteristics of prostate cancer diagnosed in Hong Kong (China) in the past two decades. Asian J Androl 2019;21:104-6.

3. Wallis CJ, Mahar A, Cheung P, et al. New rates of interventions to manage complications of modern prostate cancer treatment in older men. Eur Urol 2016;69:933-41.

4. Donovan JL, Hamdy FC, Lane JA, et al. Patient-reported outcomes after monitoring, surgery, or radiotherapy for prostate cancer. N Engl J Med 2016;375:1425-37.

5. Prabhu V, Lee T, McClintock TR, Lepor H. Short-, intermediate-, and long-term quality of life outcomes following radical prostatectomy for clinically localized prostate cancer. Rev Urol 2013;15:161-77.

6. Wei JT, Dunn RL, Litwin MS, Sandler HM, Sanda MG. Development and validation of the expanded prostate cancer index composite (EPIC) for comprehensive assessment of health-related quality of life in men with prostate cancer. Urology 2000;56:899-905.

7. Lee TK, Poon DM, Ng AC, et al. Cultural adaptation and validation of the Chinese version of the expanded prostate cancer index composite. Asia Pac J Clin Oncol 2018;14 Suppl 1:10-5.

8. Bray F, Ferlay J, Soerjomataram I, Siegel RL, Torre LA, Jemal A. Global cancer statistics 2018: GLOBOCAN estimates of incidence and mortality worldwide for 36 cancers in 185 countries. CA Cancer J Clin 2018;68:394-424.

9. Wong $\mathrm{MC}$, Goggins WB, Wang $\mathrm{HH}$, et al. Global incidence and mortality for prostate cancer: analysis of temporal patterns and trends in 36 countries. Eur Urol 2016;70:86274.

10. Lojanapiwat B, Lee JY, Gang Z, et al. Report of the third Asian Prostate Cancer Study Meeting. Prostate Int 2019;7:60-7.

11. Hamdy FC, Donovan JL, Lane JA, et al. 10-Year outcomes after monitoring, surgery, or radiotherapy for localized prostate cancer. N Engl J Med 2016;375:1415-24.

12. Nicolaisen M, Müller S, Patel HR, Hanssen TA. Quality of life and satisfaction with information after radical prostatectomy, radical external beam radiotherapy and postoperative radiotherapy: a long-term follow-up study. J Clin Nurs 2014;23:3403-14.

13. Barocas DA, Alvarez J, Resnick MJ, et al. Association between radiation therapy, surgery, or observation for localized prostate cancer and patient-reported outcomes after 3 years. JAMA 2017;317:1126-40.

14. Kakehi Y, Takegami M, Suzukamo Y, et al. Health related quality of life in Japanese men with localized prostate cancer treated with current multiple modalities assessed by a newly developed Japanese version of the Expanded Prostate Cancer Index Composite. J Urol 2007;177:185661.

15. Gordon BE, Chen RC. Patient-reported outcomes in cancer survivorship. Acta Oncol 2017;56:166-73.

16. Tyson MD, Alvarez J, Koyama T, et al. Racial variation in patient-reported outcomes following treatment for localized prostate cancer: results from the CEASAR Study. Eur Urol 2017;72:307-14. 